\title{
The impact of landmark visualization style on expert wayfinders' cognitive load during navigation
}

\author{
Armand Kapaj*, Sara Lanini-Maggi, Sara I. Fabrikant \\ Department of Geography and Digital Society Initiative, University of Zurich, Switzerland, \{armand.kapaj, sara.maggi, \\ sara.fabrikant\}@geo.uzh.ch \\ * Corresponding author
}

Keywords: Landmark visualization, mobile maps, real-world navigation study, cognitive load

\begin{abstract}
:
Our daily navigation and wayfinding activities are cognitively challenging processes, especially in unfamiliar environments (Farr et al., 2012). Landmarks have proven to facilitate navigation (Couclelis et al., 1987). Despite the acknowledged importance of landmarks for human navigation, it is still undecided how we should display them in a perceptually salient and cognitively supportive way on mobile maps. We aim to identify landmark designs that help redirect participants' gaze to the traversed environment to support participants' spatial knowledge acquisition during navigation, while also reducing participants' cognitive load.
\end{abstract}

Landmark designs at various levels of abstraction, including 2D and 3D depictions have been proposed (Döllner, 2007; Elias \& Paelke, 2008). Elias and Paelke (2008) suggest a landmark abstraction continuum for point features, ranging from $3 \mathrm{D}$ photorealistic (images) to 2D abstract (labels) on maps. Others have suggested fully photorealistic navigation displays in 3D (Nurminen \& Oulasvirta, 2008) because these seem to facilitate landmark recognition on the navigation system display, thus making the design superior to abstract 2D maps for novice users (Plesa \& Cartwright, 2008). One drawback of realistic 3D depictions is the amount of information presented to users, potentially leading to greater cognitive load and mental efforts because of the increased visual density (Liao et al., 2017). To mitigate this, Liao et al., 2017 suggest combining 2D and 3D, where only landmarks are depicted as photorealistic 3D features on the 2D mobile map.

We report on preliminary empirical results from an outdoor navigation study with expert map users and wayfinders, designed to assess how abstract 2D and more realistic 3D landmark depictions (between-subject) might influence participants' cognitive load during wayfinding in an unfamiliar environment. Based on previous inconclusive research, we formulated two competing hypotheses for this study: (1) participants' cognitive load will be higher with the more realistic 3D landmarks due to the greater amount of visual information that needs to be processed, and (2), participants' cognitive load will be lower for task-relevant 3D landmarks because their identification in the traversed environment will be facilitated by this visualization style.

We designed two mobile maps for this study (Figure 1) containing task-relevant abstract 2D building footprints (1a), and photorealistic 3D buildings (1b). The study was conducted in a residential area in Brugg, Aargau, Switzerland, unfamiliar to participants. Participants were instructed to follow a given route (approx. $1 \mathrm{~km}$ ) with the aid of the mobile map app as fast as possible, without running. They were asked to identify five landmarks in the environment, indicated along the given route (Figure 1), by raising their hand once they were next to the landmarks, to continue to reach the destination point. The buildings were chosen based on their visual and structural saliency (Sorrows \& Hirtle, 1999).

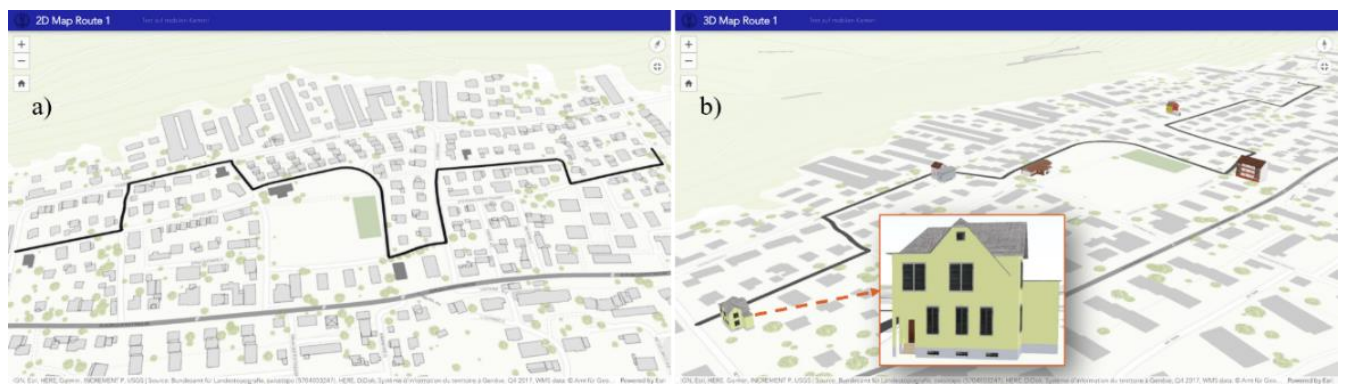

Figure 1. Landmark visualization on the interactive mobile map apps including a) abstract 2D building footprints, and b) more realistic 3D buildings (the inset view of the 3D landmark offers a zoomed-in view).

We controlled groups for gender and participants' individual differences based on their self-reported spatial strategies skills, measured with the questionnaire on spatial strategies (Münzer \& Hölscher, 2011). We also counterbalanced the starting position of the navigation task to control for landmark order effects. The map application displayed a North-up map at the start, and participants were able to interact with the map as desired (i.e., pan, zoom, rotate, and tilt). Twenty- 
two navigation experts $(\mathrm{f}=2, \mathrm{~m}=20$, average age $=37.1$ yrs., range $=24-58$ yrs. $)$ from the Engineer and Rescue Troops of the Swiss Armed Forces took part in the study. Participants were voluntarily recruited, and no incentives were provided.

Participants' brain activity was measured during navigation using a wireless 64-channel electroencephalography (EEG) device with active electrodes (LiveAmp 64 ${ }^{1}$ ), at a $500 \mathrm{~Hz}$ sampling rate. EEG data were processed in MATLAB (v9.9.0) with the EEGLAB toolbox (v2020.0). We first removed task-irrelevant (i.e., accelerometer) channels to then apply an adaptive mixture independent component (IC) analysis (AMICA; Palmer et al., 2012). We removed ICs reflecting eye and muscle movements $(\mathrm{p}>\mathrm{.90})$. We then performed a power spectrum analysis on the cleaned EEG data to compare cognitive load for the entire duration of the experiment across the 2D/3D landmark groups. For this, we relied on the power spectrum of the theta $(4-7 \mathrm{~Hz})$ and alpha $(8-13 \mathrm{~Hz})$ bands, because these are indicative of cognitive load (Klimesch, 1999), and relevant for navigation tasks (Sharma et al., 2017). Figure 2 depicts a topographic scalp density map of the EEG spectral power on the theta (2a) and alpha (2b) bands, averaged across experimental groups.
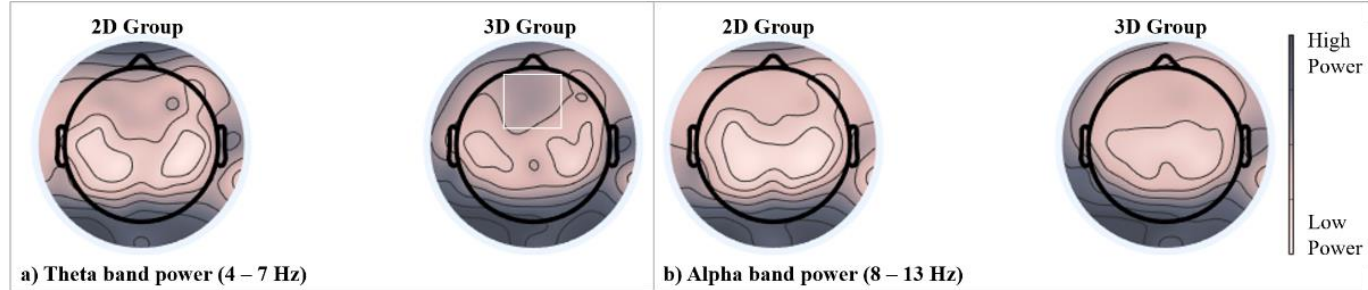

Figure 2. Similar spectral power patterns for the theta (a) and alpha (b) bands across experimental groups (2D vs. 3D).

In support of hypothesis 1, Figure 2 suggests higher theta power in the frontal lobe for the 3D group (white rectangle, in 2a) compared to the 2D group. While frontal theta power increases, and parietal alpha decreases when participants perform difficult and cognitively demanding tasks (Gevins \& Smith, 2003), we do not observe such differences in the alpha band (2b). We further compared the proportion of theta power to the total power for five task-relevant frontal electrodes (AFz, FCz, Fz, F1, and F2, see reference scalp map in Figure 3). These analyses did not yield any statistically significant difference across the 2D and 3D groups, contrary to our hypotheses.

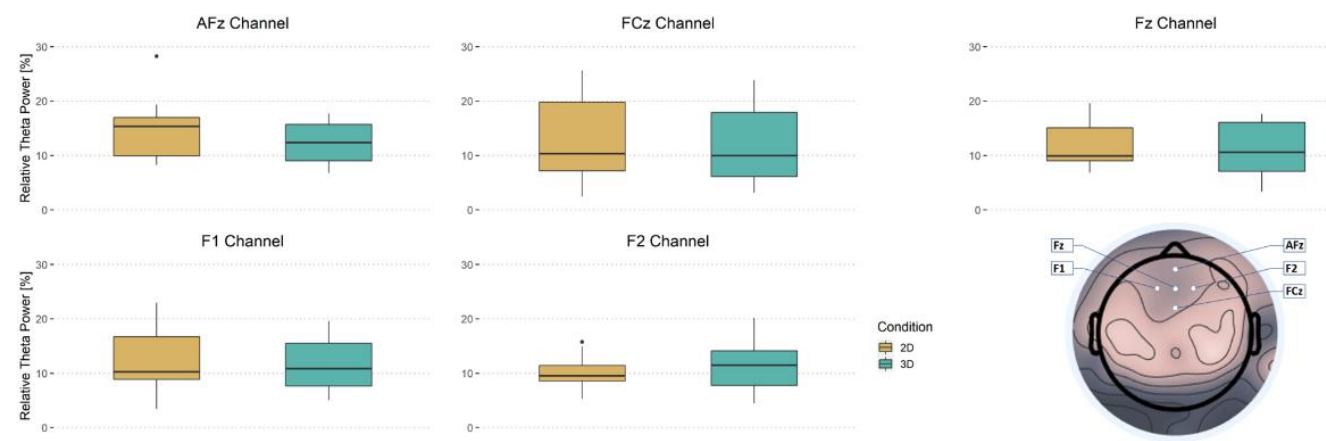

Figure 3. Relative frontal theta power on five frontal electrodes does not differ across experimental groups.

After the navigation task, participants were also asked to rate their perceived mental demand from low (0) to high (100) using the NASA Task Load Index questionnaire (Hart \& Staveland, 1988). While participants in the 2D group perceived a greater mental demand $(M d n=25.0, S D=14.7)$ compared to the $3 \mathrm{D}$ group $(M d n=20.0, S D=10.5)$, this difference is not statistically significant $(W=74.5, p=0.35, r=-0.2$ ), matching the EEG measures, but contrary to our hypotheses.

In summary, preliminary analyses on self-reports and measured EEG data reveal no statistically significant differences in cognitive load for the two tested landmark visualization styles for expert navigators. This could mean that chosen landmark visualizations do not make any difference for the cognitive load of our expert wayfinders. It could also mean that an overall power analysis averaging across the entire experiment duration covers mental load fluctuations in landmark-relevant task phases (i.e., identification, map-matching, etc.). Focusing next on frontal, central, and parietal electrodes, because these are associated with cognitive load in navigation (Wunderlich \& Gramann, 2018), we examine fluctuations of the power signals over time and then segment the EEG data into a finer temporal resolution to analyze participants' cognitive load across groups for landmark-relevant phases during navigation.

With this first EEG-supported, mobile map-assisted outdoor navigation study in cartography, we strive for high ecological validity compared to traditional laboratory navigation studies. Our research program is to develop empirically evaluated cartographic design guidelines for dynamically depicting task-relevant landmarks on mobile map displays that not only respond to wayfinders' cognitive load during navigation but also allows them to acquire spatial knowledge of the traversed environment. [References are added on a third page for completeness]

\footnotetext{
${ }^{1}$ Brain Products, Gilching, Bavaria, Germany; https://www.brainproducts.com/index.php
} 


\section{References:}

Couclelis, H., Golledge, R. G., Gale, N., \& Tobler, W. (1987). Exploring the anchor-point hypothesis of spatial cognition. Journal of Environmental Psychology, 7(2), 99-122. https://doi.org/10.1016/S0272-4944(87)80020-8

Döllner, J. (2007). Non-Photorealistic 3D Geovisualization. In Multimedia Cartography (pp. 229-240). https://doi.org/10.1007/978-3-540-36651-5_16

Elias, B., \& Paelke, V. (2008). User-Centered design of landmark visualizations. In Lecture Notes in Geoinformation and Cartography (pp. 33-56). https://doi.org/10.1007/978-3-540-37110-6_3

Farr, A. C., Kleinschmidt, T., Yarlagadda, P., \& Mengersen, K. (2012). Wayfinding: A simple concept, a complex process. Transport Reviews, 32(6), 715-743. https://doi.org/10.1080/01441647.2012.712555

Gevins, A., \& Smith, M. E. (2003). Neurophysiological measures of cognitive workload during human-computer interaction. Theoretical Issues in Ergonomics Science, 4(1-2), $113-131$. https://doi.org/10.1080/14639220210159717

Hart, S. G., \& Staveland, L. E. (1988). Development of NASA-TLX (Task Load Index): Results of Empirical and Theoretical Research. Advances in Psychology, 52, 139-183. https://doi.org/10.1016/S0166-4115(08)62386-9

Klimesch, W. (1999). EEG alpha and theta oscillations reflect cognitive and memory performance: a review and analysis. Brain Research Reviews, 29(2-3), 169-195. https://doi.org/10.1016/S0165-0173(98)00056-3

Liao, H., Dong, W., Peng, C., \& Liu, H. (2017). Exploring differences of visual attention in pedestrian navigation when using 2D maps and 3D geo-browsers. Cartography and Geographic Information Science, 44(6), 474-490. https://doi.org/10.1080/15230406.2016.1174886

Münzer, S., \& Hölscher, C. (2011). Entwicklung und Validierung eines Fragebogens zu räumlichen Strategien. Diagnostica, 57(3), 111-125. https://doi.org/10.1026/0012-1924/a000040

Nurminen, A., \& Oulasvirta, A. (2008). Designing Interactions for Navigation in 3D Mobile Maps. In Lecture Notes in Geoinformation and Cartography (pp. 198-227). https://doi.org/10.1007/978-3-540-37110-6_10

Palmer, J. A., Kreutz-Delgado, K., \& Makeig, S. (2012). AMICA: An adaptive mixture of independent component analyzers with shared components. Swartz Center for Computatonal Neursoscience, University of California San Diego, Tech. Rep.

Plesa, M. A., \& Cartwright, W. (2008). Evaluating the Effectiveness of Non-Realistic 3D Maps for Navigation with Mobile Devices. In Map-based Mobile Services (pp. 80-104). https://doi.org/10.1007/978-3-540-37110-6_5

Sharma, G., Gramann, K., Chandra, S., Singh, V., \& Mittal, A. P. (2017). Brain connectivity during encoding and retrieval of spatial information: individual differences in navigation skills. Brain Informatics, 4(3), $207-217$. https://doi.org/10.1007/s40708-017-0066-6

Sorrows, M. E., \& Hirtle, S. C. (1999). The Nature of Landmarks for Real and Electronic Spaces. In International Conference on Spatial Information Theory (pp. 37-50). https://doi.org/10.1007/3-540-48384-5_3

Wunderlich, A., \& Gramann, K. (2018). Electrocortical evidence for long-term incidental spatial learning through modified navigation instructions. In Spatial Cognition XI (Vol. 11034, pp. 261-278). https://doi.org/10.1007/9783-319-96385-3_18 